\title{
Coordinated MultiPoint Uplink Capacity over a MIMO Composite Fading Channel
}

\author{
Symeon Chatzinotas*, Björn Ottersten ${ }^{* \dagger}$ \\ ${ }^{*}$ SnT - securityandtrust.lu, University of Luxembourg, Email: \{Symeon.Chatzinotas, Bjorn.Ottersten\}@uni.lu \\ ${ }^{\dagger}$ Royal Institute of Technology (KTH), Sweden, Email: bjorn.ottersten@ee.kth.se
}

\begin{abstract}
Coordinated Multipoint comprises a set of communication strategies for cellular systems which are based on cooperation/coordination between Base Stations (BSs). These strategies have been recently included in LTE standardization in order to meet the spectral efficiency requirements. In this paper, the focus is on the uplink capacity of a cellular system over a composite fading channel, which combines Ricean fading and shadowing. In order to realistically model the cellular system, path loss and distributed users are also considered. Finally, the channel model incorporates multiple antennas, as dictated by the latest wireless standards. Based on random matrix and free probability theory, closed-form expressions are derived for the channel capacity and the MMSE achievable rate. In this context, strong Line-of-Sight component is shown to degrade capacity performance, while large shadowing statistics benefit it.
\end{abstract}

\section{INTRODUCTION}

Intercell interference is the main impairment in cellular communications and tackling it is considered as the final frontier in optimizing cellular systems. Most of the approaches in the past have been focused in avoiding (e.g. orthogonalization) or mitigating (e.g. beamforming) intercell interference. However, recently a number of communication strategies have been proposed in order to exploit intercell signals cooperation/coordination between Base Stations (BSs). These strategies have been recently included in LTE-Advanced standardization in order to meet the spectral efficiency requirements and are known under the term Coordinated MultiPoint (CoMP) [1].

In this paper, the uplink capacity is studied for a cellular system which employs multicell joint decoding, one of the CoMP techniques that require $\mathrm{BS}$ cooperation. The main improvement over existing literature is the incorporation of a composite fading channel, which combines Ricean fading and shadowing. Furthermore, in order to realistically model the cellular system, path loss and distributed users are also considered. Finally, the channel model incorporates multiple antennas, as dictated by the latest wireless standards, such as LTE and WiMax. In this context, closed-form expressions are derived for the channel capacity and the MMSE achievable rate, based on random matrix and free probability theory.

In the remainder of this paper, an overview of related work is presented in section II. In section III, the considered channel model is presented and in section IV the capacity performance of the CoMP uplink is derived for optimal and MMSE multicell receivers. In section $\mathrm{V}$, the capacity performance is quantified through numerical simulations and section VI concludes the paper.

\section{A. Notation}

Throughout the formulations of this paper, $\mathbb{E}[\cdot]$ denotes the expectation, $\mathbb{V}[\cdot]$ denotes the variance, $(\cdot)^{\dagger}$ denotes the conjugate transpose matrix, $(\cdot)^{T}$ denotes the transpose matrix, $\odot$ denotes the Hadamard product and $\otimes$ denotes the Kronecker product.The Frobenius norm of a matrix or vector is denoted by $\|\cdot\| . \mathbf{I}_{n}$ denotes a $n \times n$ identity matrix, $\mathbb{I}_{n \times m}$ a $n \times m$ matrix of ones, $\mathbf{1}_{n}$ a $n \times 1$ vector of ones and $\mathbf{0}$ a zero matrix.

\section{Preliminaries \& Related Work}

\section{A. Coordinated MultiPoint}

Coordinated Multi-Point (CoMP) transmission/reception is a term employed by 3GPP LTE-A standardization initiative in order to describe a group of advanced multi-cell coordination techniques [1]. In this paper, we focus on Multicell Joint Processing (MJP) in the uplink channel, where user signals received by multiple $\mathrm{BSs}$ are jointly decoded in order to mitigate inter-cell interference. This MJP scenario has appeared in the literature under various research topics, such as BS cooperation, Network MIMO and Distributed Antenna Systems (DAS). Its sum-rate capacity has been studied for optimal and MMSE receivers [2], as well as for various channel impairments, such as path loss [3], shadowing [4], Rayleigh/Rice fading [5], [6] and antenna correlation [2], [7]. In this paper, we consider both optimal and MMSE receivers for an MJP model incorporating spatially distributed users, path loss, Ricean fading, shadowing and multiple antennas.

\section{B. Composite Fading Channel}

In terrestrial cellular systems, the effect of channel fading can be classified as fast (multipath) or slow (shadowing). Fast fading is due to combination of many signal replicas arriving through different paths, while slow fading is due to large obstructions between the receiver and the transmitter line of sight. In MJP literature, multipath and shadowing have been studied separately but little is known about their combination. In this direction, this paper considers a composite fading channel which combines Ricean fading with shadowing for an MJP model and studies their effect on the capacity performance. In existing literature, composite fading channels have been studied for the uplink of distributed MIMO systems taking into account a range of microscopic-macroscopic fading 


$$
\mathbf{y}_{n}=\sum_{m=1}^{M} \sqrt{\alpha_{n, m} \xi_{n, m}}\left(\sqrt{\frac{K}{K+1}} \mathbf{L}_{n, m}+\sqrt{\frac{1}{K+1}} \mathbf{G}_{n, m}\right) \mathbf{x}_{m}+\mathbf{z}_{n},
$$

combinations such as Rayleigh-lognormal MIMO [8], [9] Nakagami-lognormal MIMO [10] Nakagami-Gamma SIMO [11] Nakagami-Gamma MIMO [12]. In the aforementioned work, the usual performance metric is the ergodic channel capacity [13], [8], [10], [12], [9], while outage capacity is considered in [11], [14]. Furthermore, the effect of Kronecker correlated antennas has been considered in [15], [14], while the downlink of distributed antenna systems in [16], [13].

Nevertheless, all previous work assumes that a single user is served by a number of BSs and this entails a channel matrix which can be written as the product of a full rank fading matrix with a diagonal shadowing matrix. In this paper, multiple users are served by multiple BSs and therefore this channel modelling no longer applies. In addition, a line-ofsight component is assumed and as a result the channel is Ricean-lognormal MIMO or Ricean-Gamma MIMO. Finally, both optimal and linear MMSE receivers are considered. A composite MJP channel has been already studied in [4], but it focuses only on optimal capacity for the Ricean-lognormal case.

\section{System \& Channel Model}

The considered system comprises a planar hexagonal multicell system with $N$ BSs (eNBs according to 3GPP) and $M \geq N$ users (UEs according to 3GPP). Each BS is equipped with $r$ omni antennas ${ }^{1}$, while each user with $t$ omni antennas ${ }^{2}$. The multicell system employs full frequency reuse and the transmission strategy is superposition coding with Gaussian input symbols over a flat-faded memoryless channel.

Under these assumptions, the $r \times 1$ received signal vector at the $n$th BS is given by eq. (1), where $\mathbf{x}_{m}$ is the $t \times 1$ transmit signal vector of the $m$ th user with $\mathbb{E}\left[\mathbf{x}_{m} \mathbf{x}_{m}^{\dagger}\right]=\gamma \mathbf{I}$ and $\mathbf{z}_{m}$ is the Additive White Gaussian Noise (AWGN) at the receiver with $\mathbb{E}\left[\mathbf{z}_{m} \mathbf{z}_{m}^{\dagger}\right]=\mathbf{I}$. The coefficient $\alpha_{n, m}$ is real and deterministic modelling the path loss, while the coefficient $\xi_{n, m}$ is real and random $\left(\mathbb{E}\left[\xi_{n, m}\right]=\mu_{\xi}, \mathbb{V}\left[\xi_{n, m}\right]=\sigma_{\xi}^{2}\right)$ modelling the shadowing (macroscopic fading) ${ }^{3}$. In addition, $K$ is the Ricean factor determining the power split between the Line-of-Sight (LoS) $\mathbf{L}_{n, m}$ and non-LoS component $\mathbf{G}_{n, m}$. The $r \times t$ matrix $\mathbf{L}_{n, m}$ is assumed to be deterministic and unitrank $\left.\operatorname{rank}\left(\mathbf{L}_{n, m}\right)=1\right)$ due to spatial correlation of the LoS component with normalization $\operatorname{trace}\left(\mathbf{L}_{n, m} \mathbf{L}_{n, m}^{\dagger}\right)=r$. The $r \times t$ matrix $\mathbf{G}_{n, m}$ is assume to be random due to microscopic

\footnotetext{
${ }^{1}$ Sectorization is not considered since in CoMP systems with coherent joint processing it is beneficial to receive the transmitted signals in multiple BS antennas .

${ }^{2}$ No CSI is available at transmit side and thus no input optimization is considered.

${ }^{3}$ The shadowing coefficients are assumed to be identically distributed for simplicity. The presented analysis is valid and easily extendable for non-identically distributed shadowing coefficients. Since shadowing is slow varying even longer codes are required to achieve Shannon capacity,
}

fading with independent identically distributed (i.i.d.) complex circularly symmetric (c.c.s.) elements of zero mean and unit variance.

In order to model the received signal vector across the whole multicell system, all received signal vectors $\mathbf{y}_{n}$ can be accumulated in:

$$
\mathbf{y}=\mathbf{H} \mathbf{x}+\mathbf{z}
$$

where $\mathbf{y}=\left[\mathbf{y}_{1}^{T} \ldots \mathbf{y}_{N}^{T}\right]^{T}, \mathbf{x}=\left[\mathbf{x}_{1}^{T} \ldots \mathbf{x}_{M}^{T}\right]^{T}, \mathbf{z}=\left[\mathbf{z}_{1}^{T} \ldots \mathbf{z}_{N}^{T}\right]^{T}$ and

$$
\mathbf{H}=\left((\mathbf{A} \odot \boldsymbol{\Xi}) \otimes \mathbb{I}_{r \times t}\right) \odot\left(\sqrt{\frac{K}{K+1}} \mathbf{L}+\sqrt{\frac{1}{K+1}} \mathbf{G}\right),
$$

where $\mathbf{A}$ and $\boldsymbol{\Xi}$ are $N \times M$ matrices aggregating the square roots of path loss $\alpha_{n, m}$ and shadowing $\xi_{n, m}$ coefficients respectively, while and $\mathbf{G}$ are $N r \times M t$ matrices aggregating the $\operatorname{LoS} \mathbf{L}_{n, m}$ and fading $\mathbf{G}_{n, m}$ coefficients respectively.

\section{CApacity Performance}

The channel capacity and achievable rates in multiuser MIMO systems is greatly dependent on the eigenvalue distribution of the sample covariance matrix $\mathbf{H H}^{\dagger}$ of the aggregate channel matrix. In this direction, the eigenvalue distribution is analytically derived based on principles from random matrix and free probability theory and subsequently is exploited in order to find closed-form approximations for the channel capacity and the MMSE achievable rate.

\section{A. Eigenvalue Distribution}

Since the channel matrix involves random variables, the corresponding eigenvalue of $\mathbf{H H}^{\dagger}$ would also be random. However, asymptotic analysis has shown that for large matrix dimensions the eigenvalue distribution becomes deterministic. More importantly, this deterministic solution holds well even for finite dimensions. In this direction, the following derivations are performed for the regime where $r, t \rightarrow \infty$ with $\frac{t}{r}=\beta$.

1) Preliminaries: The following paragraphs describe some basic definitions and theorems which will be used for the derivations.

Definition 4.1: The asymptotic cumulative distribution function (a.e.p.d.f.) of the eigenvalues of matrix $\lambda_{i}\left(\mathbf{H H}^{\dagger}\right)$ is defined as

$$
\mathrm{F}_{\mathbf{H H}^{\dagger}}^{\infty}(x)=\lim _{n \rightarrow \infty} \frac{1}{n} \sum_{i=1}^{n} 1\left\{\lambda_{i}\left(\mathbf{H H}^{\dagger}\right) \leq x\right\}
$$

and the corresponding asymptotic eigenvalue probability distribution function

$$
\mathrm{f}_{\mathbf{H} \mathbf{H}^{\dagger}}^{\infty}(x)=\mathrm{dF}_{\mathbf{H} \mathbf{H}^{\dagger}}^{\infty}(x) / \mathrm{d} x .
$$


Theorem 4.1: The a.e.p.d.f. $\frac{1}{r} \mathbf{G}^{\dagger} \mathbf{G}$ converges almost surely (a.s.) to the non-random limiting eigenvalue distribution of the Marčenko-Pastur law [17], whose Shannon transform with parameter $y$ is given by

$$
\begin{aligned}
\mathcal{V}_{\frac{1}{N r} \mathbf{G}^{\dagger} \mathbf{G}}(u) & \stackrel{a . s}{\longrightarrow} \mathcal{V}_{\mathrm{MP}}(u, v) \\
\mathcal{V}_{\mathrm{MP}}(u, v) & =\log \left(1+u-\frac{1}{4} \phi(u, v)\right) \\
& +\frac{1}{v} \log \left(1+u v-\frac{1}{4} \phi(u, v)\right)-\frac{1}{4 u v} \phi(u, v) \\
\phi(u, v) & =\left(\sqrt{u(1+\sqrt{v})^{2}+1}-\sqrt{u(1-\sqrt{v})^{2}+1}\right)^{2}
\end{aligned}
$$

and $\eta$-transform with parameter $y$ is given by

$$
\eta_{\mathrm{MP}}(u, v)=1-\frac{\phi(u, v)}{4 u v}
$$

where $v$ is the ratio of the horizontal to the vertical dimension of the $\mathbf{G}$ matrix.

2) Derivation: The following theorems outline the derivation of a.e.p.d.f. of $\frac{1}{N r} \mathbf{H H}^{\dagger}$.

Theorem 4.2: The a.e.p.d.f. of $\frac{1}{N r} \mathbf{H H}^{\dagger}$ is equivalent to the a.e.p.d.f. of $\frac{1}{N r} \overline{\mathbf{H}} \overline{\mathbf{H}}^{\dagger}$ with

$$
\overline{\mathbf{H}}=\left((\mathbf{A} \odot \boldsymbol{\Xi}) \otimes \mathbb{I}_{r \times t}\right) \odot\left(\sqrt{\frac{1}{K+1}} \mathbf{G}\right) .
$$

Proof: Since $\operatorname{rank}\left(\mathbf{L}_{n, m}\right)=1, \operatorname{rank}(\mathbf{L})=N$. For $r, t \rightarrow \infty, \operatorname{rank}(\mathbf{L}) / r \rightarrow 0$ and therefore the contribution of the Ricean components in the a.e.p.d.f. of $\frac{1}{N r} \mathbf{H} \mathbf{H}^{\dagger}$ becomes marginal.

Theorem 4.3: The a.e.p.d.f. of $\frac{\gamma}{N r} \tilde{\mathbf{H}} \tilde{\mathbf{H}}^{\dagger}$ where

$$
\tilde{\mathbf{H}}=\left(\boldsymbol{\Xi} \otimes \mathbb{I}_{r \times t}\right) \odot\left(\sqrt{\frac{1}{K+1}} \mathbf{G}\right)
$$

converges almost surely to the Marčenko-Pastur law with $u=$ $\gamma\left(\mu_{\xi}^{2}+\sigma_{\xi}^{2}\right) / 4$ and $v=M \beta / N$.

Proof: Since the random elements of $\boldsymbol{\Xi}$ and $\mathbf{G}$ are independent, the elements of $\tilde{\mathbf{H}}$ will be independent and identically distributed with $\mathbb{E}\left[\sqrt{\xi_{n, m}}\right]=\mu_{\xi} / 2, \mathbb{V}\left[\sqrt{\xi_{n, m}}\right]=\sigma_{\xi}^{2} / 4$, $\mathbb{E}\left[\tilde{h}_{n, m}\right]=\mathbb{E}\left[\sqrt{\xi_{n, m}}\right] \mathbb{E}\left[g_{n, m}\right]=0$ and

$$
\begin{array}{rlr}
\mathbb{V}\left[\tilde{h}_{n, m}\right]=\left(\mathbb{E}\left[\sqrt{\xi_{n, m}}\right]\right)^{2} \mathbb{V}\left[g_{n, m}\right] & +\left(\mathbb{E}\left[g_{n, m}\right]\right)^{2} \mathbb{V}\left[\sqrt{\xi_{n, m}}\right] \\
& +\mathbb{V}\left[g_{n, m}\right] \mathbb{V}\left[\sqrt{\xi_{n, m}}\right] \\
=\left(\mu_{\xi}^{2}+\sigma_{\xi}^{2}\right) / 4 . &
\end{array}
$$

Thus, the Marčenko-Pastur law still applies for the a.e.p.d.f. of $\tilde{\mathbf{H}} \tilde{\mathbf{H}}^{\dagger}$, but with variance $\mathbb{V}\left[\tilde{h}_{n, m}\right]$.

Theorem 4.4: The a.e.p.d.f. of $\frac{\gamma}{N r} \overline{\mathbf{H}} \overline{\mathbf{H}}^{\dagger}$ can be approximated by a scaled version of the Marčenko-Pastur law with

$$
y=\gamma \frac{\|\mathbf{A}\|^{2}}{M N(K+1)} \frac{\mu_{\xi}^{2}+\sigma_{\xi}^{2}}{4}
$$

and $v=M \beta / N$.

Proof: Based on the free probability approximation presented in [2], the effect of the path loss (variance profile) matrix will result in a scaled version of the Marčenko-Pastur law.

\section{B. Channel Capacity}

The channel capacity can be expressed in terms of the derived a.e.p.d.f. as follows:

$$
\begin{aligned}
C_{\mathrm{opt}} & =\lim _{r, t \rightarrow \infty} \frac{1}{N r} \mathcal{I}(\mathbf{x} ; \mathbf{y} \mid \mathbf{H}) \\
& =\lim _{r, t \rightarrow \infty} \frac{1}{N r} \mathbb{E}\left[\log \operatorname{det}\left(\mathbf{I}+\gamma \mathbf{H H}^{\dagger}\right)\right] \\
& =\lim _{r, t \rightarrow \infty} \mathbb{E}\left[\frac{1}{N r} \sum_{i=1}^{N r} \log \left(1+\tilde{\gamma} \lambda_{i}\left(\frac{1}{N r} \mathbf{H H}^{\dagger}\right)\right)\right] \\
& =\int_{0}^{\infty} \log (1+\tilde{\gamma} x) \mathrm{f}_{\frac{1}{N r}}^{\infty} \mathbf{H H}^{\dagger}(x) \mathrm{d} x \\
& =\mathcal{V}_{\frac{1}{N r}} \mathbf{H H}^{\dagger}(\tilde{\gamma})=\frac{M \beta}{N} \mathcal{V}_{\frac{1}{N r}} \mathbf{H}^{\dagger} \mathbf{H}(\tilde{\gamma}) \\
& \simeq \frac{M \beta}{N} \mathcal{V}_{\mathrm{MP}}\left(\frac{\|\mathbf{A}\|^{2}}{M N(K+1)} \frac{\mu_{\xi}^{2}+\sigma_{\xi}^{2}}{4} \tilde{\gamma}, \frac{M \beta}{N}\right)
\end{aligned}
$$

where $\tilde{\gamma}=N r \gamma^{4}$.

\section{MMSE Linear Filtering}

A bound on the achievable rate after MMSE linear filtering and single-user decoding can be expressed in terms of the derived a.e.p.d.f. as in (16).

\section{Numerical Results}

This section presents a number of numerical results in order to verify the accuracy of the derived analytical expressions and evaluate the performance under optimal joint decoding (channel capacity) and MMSE linear filtering followed by single-user decoding. In the following figures, points represent values calculated through Monte Carlo simulations, while lines refer to curves evaluated based on the analytical expressions of section IV. More specifically, the simulations are performed by generating $10^{3}$ instances of random Gaussian matrices $\mathbf{G}_{n, m}$, each one representing a single fading realization of the system. Each LoS matrix $\mathbf{L}_{n, m}$ is produced using the product $\mathrm{ll}^{\dagger}$ of a randomly generated vector 1 which is assumed to be fixed for all fading realizations. In addition, the path loss coefficients $\alpha_{n, m}$ are generated using a power law model and random user positions. Finally, the shadowing coefficients $\xi_{n, m}$ are drawn from a lognormal distribution with density function:

$$
f_{X}(\xi ; \mu, \sigma)=\frac{1}{\xi \sigma \sqrt{2 \pi}} e^{-\frac{(\ln \xi-\mu)^{2}}{2 \sigma^{2}}},
$$

mean $\mathbb{E}[\xi]=e^{\mu+\sigma^{2} / 2}$ and variance $\mathbb{V}[\xi]=\left(e^{\sigma^{2}}-1\right) e^{2 \mu+\sigma^{2}}$. It should be noted that the results are straightforwardly extendable for shadowing drawn from the Gamma distribution with density function:

$$
f(\xi ; k, \theta)=\xi^{k-1} \frac{e^{-\xi / \theta}}{\theta^{\xi} \Gamma(k)},
$$

mean $\mathbb{E}[\xi]=k \theta$ and variance $\mathbb{V}[\xi]=k \theta^{2}$ or any other applicable distribution by adapting $\mathbb{E}[\xi]$ and $\mathbb{V}[\xi]$.

${ }^{4}$ The variable $\tilde{\gamma}$ is kept finite while $r \rightarrow \infty$ so that the system power is bounded. 


$$
\begin{aligned}
C_{\mathrm{mmse}} & =\lim _{r, t \rightarrow \infty} \frac{M \beta}{N} \mathbb{E}\left[\log \left(\frac{1}{M t} \sum_{k=1}^{M t}\left(1+\operatorname{SINR}_{k}\right)\right)\right] \\
& =-\lim _{r, t \rightarrow \infty} \frac{M \beta}{N} \mathbb{E}\left[\log \left(\frac{1}{M t} \sum_{k=1}^{M t}\left[\left(\mathbf{I}_{M t}+\gamma \mathbf{H}^{\dagger} \mathbf{H}\right)^{-1}\right]_{k, k}\right)\right] \\
& \leq-\lim _{r, t \rightarrow \infty} \frac{M \beta}{N} \log \left(\frac{1}{M t} \mathbb{E}\left[\operatorname{Tr}\left\{\left(\mathbf{I}_{M t}+\gamma \mathbf{H}^{\dagger} \mathbf{H}\right)^{-1}\right\}\right]\right) \\
& =-\lim _{N \rightarrow \infty} \frac{M \beta}{N} \log \left(\mathbb { E } \left[\frac{1}{M t} \sum_{k=1}^{M t} \frac{1}{\left.\left.1+\tilde{\gamma} \lambda_{k}\left(\frac{1}{N r} \mathbf{H}^{\dagger} \mathbf{H}\right)\right]\right)}\right.\right. \\
& =-\frac{M \beta}{N} \log \left(\int_{0^{+}}^{\infty} \frac{1}{1+\tilde{\gamma} x} f_{\frac{1}{N r} \mathbf{H}^{\dagger} \mathbf{H}}(x) d x\right)=-\frac{M \beta}{N} \log \left(\eta_{\frac{1}{N r} \mathbf{H}^{\dagger} \mathbf{H}}(\tilde{\gamma})\right) \\
& =-\frac{M \beta}{N} \log \left(\eta_{\mathrm{MP}}\left(\frac{\|\mathbf{A}\|^{2}}{M N(K+1)} \frac{\mu_{\xi}^{2}+\sigma_{\xi}^{2}}{4} \tilde{\gamma}, \frac{M \beta}{N}\right)\right)
\end{aligned}
$$

TABLE I

PARAMETERS FOR CAPACITY RESULTS

\begin{tabular}{l|c|c} 
Parameter & Symbol & Value \\
\hline Number of BS & $N$ & 7 \\
Number of UTs & $M$ & 28 \\
Antennas per BS & $r$ & 8 \\
Antennas per UT & $t$ & 2,8 \\
UT Transmit Power & & $200 m W$ \\
Rice factor & $K$ & $0-100$ \\
Shadowing mean & $\mu_{\xi}$ & $1-10$ \\
Shadowing variance & $\sigma_{\xi}^{2}$ & $1-10$ \\
Path loss exponent & $\eta$ & 3.45 \\
Cell radius & $R$ & $0.1-1 K m$ \\
Number of MC iterations & & $10^{3}$ \\
\hline
\end{tabular}

Subsequently, the per-cell capacities are evaluated by averaging over the system realizations using: a) eq. (13) for channel capacity, b) eq. (15) for MMSE filtering. In parallel, the analytical curves are evaluated based on: a) eq. (14) for channel capacity, b) eq. (16) for MMSE filtering. Table I presents an overview of the parameter values and ranges used for producing the numerical results of the figures. The percell sum-rates are plotted versus the cell radius, capturing a wide range of cellular systems, e.g. femtocells, microcells, macrocells etc. It should be noted that in the presented results we have chosen $t=8$ for optimal decoding, while $t=2$ for MMSE. This is because the MMSE performance is greatly degraded due to rank deficiency, if the total number of transmit antennas $N r$ is larger that that of the receive antennas $M t$.

As it can be seen in fig. 1 and 3, there is a very good agreement of analytical and simulated results. Increasing $K$ namely the power of the LoS component, while keeping the total channel power fixed, degrades both optimal and MMSE sum-rate. However, this negative effect is even more pronounced in the optimal case.

In fig. 2 and 4, a quite good agreement can be seen while simultaneously varying the mean and variance of the shadowing coefficients. The main observation is that shadowing can be beneficial for both optimal and MMSE sum-rates and this

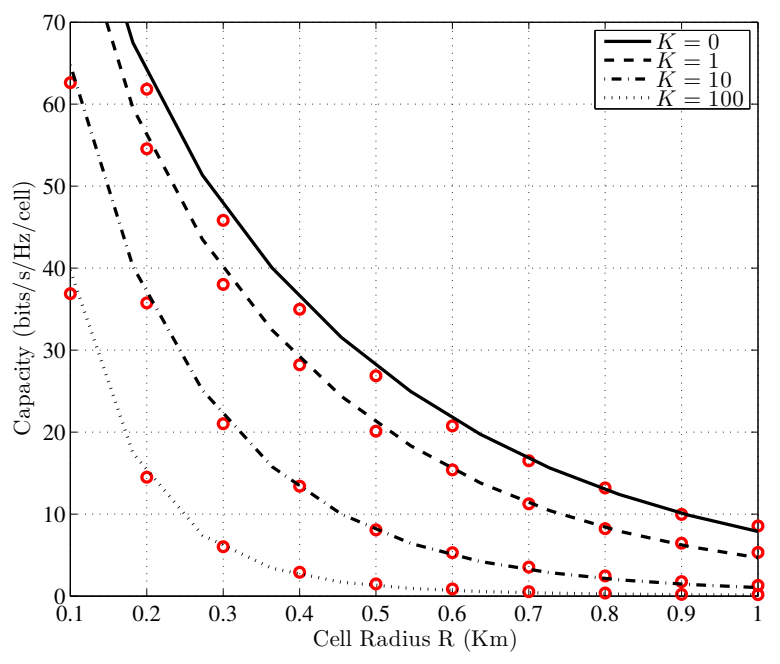

Fig. 1. Per-cell channel sum-rate capacity scaling versus the Ricean $K$-factor. Stronger LoS degrades performance. Parameters: $\mu_{\xi}=\sigma_{\xi}^{2}=2, t=8$.

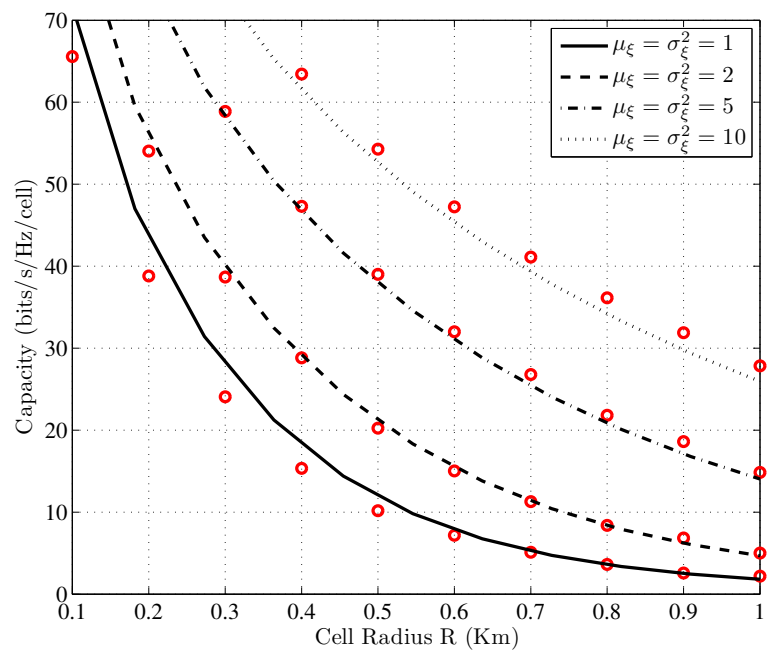

Fig. 2. Per-cell channel sum-rate capacity scaling versus shadowing statistics. Stronger shadowing benefits performance. Parameters: $K=1, t=8$. 


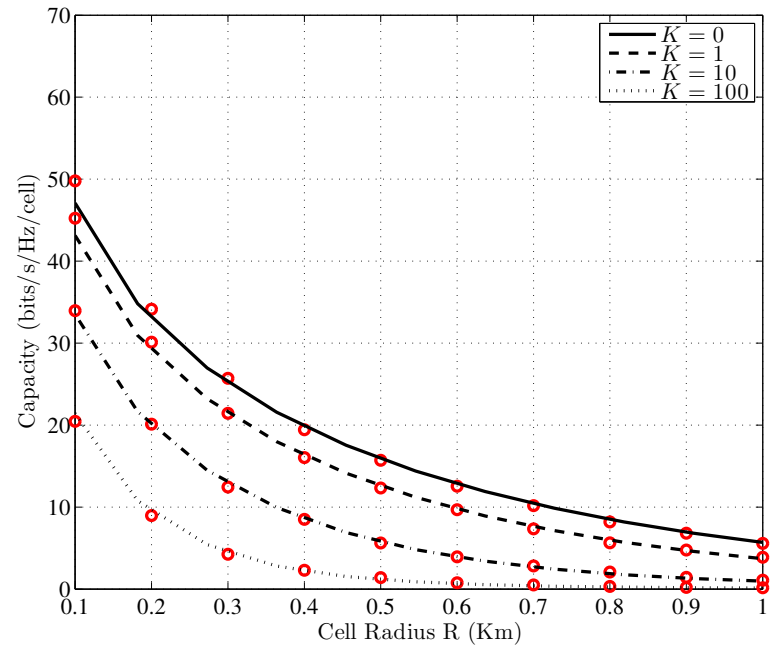

Fig. 3. Per-cell MMSE sum-rate scaling versus the Ricean $K$-factor. Stronger LoS degrades performance. Parameters: $\mu_{\xi}=\sigma_{\xi}^{2}=2, t=2$.

is inline with the beneficial effect of fading due to multiuser diversity as observed in [5]. However, it should be noted that in this comparison the channel power is increased by increasing the shadowing statistics unlike [4] where a sum-rate loss is observed.

\section{Conclusions}

In this paper, the uplink of a Coordinated MultiPoint system was studied in terms of sum-rate under optimal joint decoding and MMSE linear filtering. The novelty lies in the consideration of a MIMO composite channel which combines the effects of shadowing, Ricean fast fading and asymmetric multiuser channels due to geographical distribution and path loss. In this setting, analytical approximations based on free probability and random matrix theory were derived and verified via Monte Carlo simulation. The main outcome of the study is that low Ricean factors and strong shadowing statistics can be beneficial for the sum-rate. In other words, varying channels outperform fixed AWGN channels in terms of ergodic sumrate.

\section{ACKNOWLEDGMENT}

This work was partially supported by the National Research Fund, Luxembourg under the CORE project "CO${ }^{2} \mathrm{SAT}$ : Cooperative and Cognitive Architectures for Satellite Networks".

\section{REFERENCES}

[1] 3GPP, "Further advancements for E-UTRA physical layer aspects," 3rd Generation Partnership Project, Tech. Rep. TR 36.814.

[2] S. Chatzinotas, M. Imran, and R. Hoshyar, "On the multicell processing capacity of the cellular MIMO uplink channel in correlated Rayleigh fading environment," IEEE Trans. Wireless Commun., vol. 8, no. 7, pp. 3704-3715, July 2009.

[3] S. Chatzinotas, M. Imran, and C. Tzaras, "On the capacity of variable density cellular systems under multicell decoding," IEEE Commun. Lett., vol. 12, no. 7, pp. 496 - 498, Jul 2008.

[4] D. Kaltakis, M. Imran, and C. Tzaras, "Information theoretic capacity of cellular multiple access channel with shadow fading," IEEE Trans. Commun., vol. 58, no. 5, pp. $1468-1476$, may 2010.

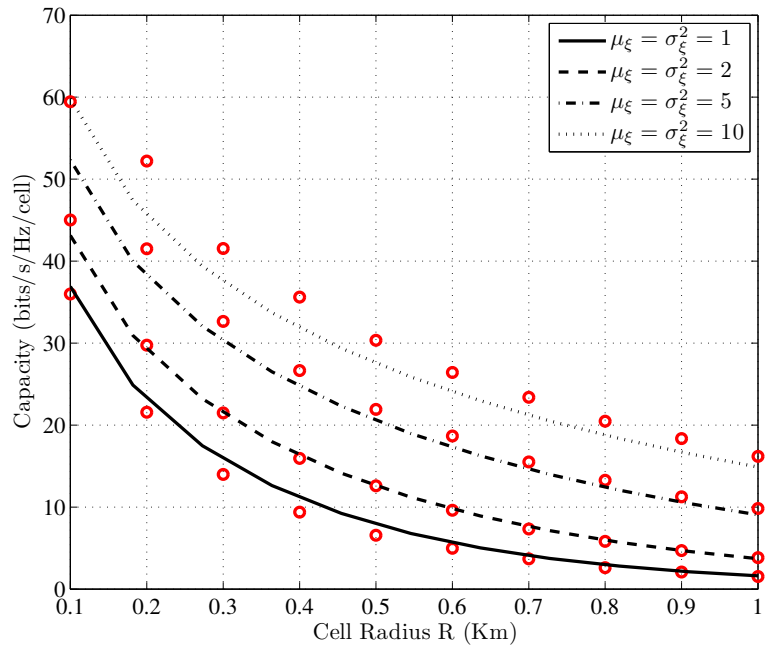

Fig. 4. Per-cell MMSE sum-rate scaling versus shadowing statistics. Stronger shadowing benefits performance. Parameters: $K=1, t=2$.

[5] O. Somekh and S. Shamai, "Shannon-theoretic approach to a Gaussian cellular multiple-access channel with fading," IEEE Trans. Inf. Theory, vol. 46, no. 4, pp. 1401-1425, Jul 2000.

[6] J. N. J. Hoydis, A. Kammou and M. Debbah, "Outage performance of cooperative small cell systems under Rician fading channels," in IEEE International Workshop on Signal Processing Advances for Wireless Communications (SPAWC'11), San Francisco, CA, US, Jun. 2011.

[7] R. Couillet, M. Debbah, and J. W. Silverstein, "A deterministic equivalent for the capacity analysis of correlated multi-user MIMO channels," CoRR, vol. abs/0906.3667, 2009.

[8] W. Roh and A. Paulraj, "MIMO channel capacity for the distributed antenna systems," in IEEE 56th Vehicular Technology Conference (VTC 2002-Fall), vol. 2, 2002, pp. $706-709$.

[9] D. Wang, X. You, J. Wang, Y. Wang, and X. Hou, "Spectral efficiency of distributed MIMO cellular systems in a composite fading channel," in IEEE International Conference on Communications (ICC '08), May 2008, pp. $1259-1264$.

[10] C. Zhong, K.-K. Wong, and S. Jin, "Capacity bounds for MIMO Nakagami- m fading channels," IEEE Trans. Signal Processing, vol. 57, no. 9, pp. $3613-3623$, sept. 2009.

[11] S. Al-Ahmadi and H. Yanikomeroglu, "The ergodic and outage capacities of distributed antenna systems in generalized-K fading channels," in IEEE 21st International Symposium on Personal Indoor and Mobile Radio Communications (PIMRC'10), sept. 2010, pp. $662-666$.

[12] M. Matthaiou, N. Chatzidiamantis, G. Karagiannidis, and J. Nossek, "On the capacity of generalized- K fading MIMO channels," IEEE Trans. Signal Processing, vol. 58, no. 11, pp. 5939 -5944, nov. 2010.

[13] J.-Y. Wang, J.-B. Wang, X.-Y. Dang, M. Lin, Y. Jiao, and M. Chen, "System capacity analysis of downlink distributed antenna systems over composite channels," in 12th IEEE International Conference on Communication Technology (ICCT'10), nov. 2010, pp. 1076-1079.

[14] H. Dai, H. Zhang, and Q. Zhou, "Some analysis in distributed MIMO systems," Journal of Communications, vol. 2, no. 3, pp. 43-50, 2007.

[15] M. Matthaiou, N. Chatzidiamantis, and G. Karagiannidis, "A new lower bound on the ergodic capacity of distributed MIMO systems," IEEE Signal Processing Letters, vol. 18, no. 4, pp. 227 -230, april 2011.

[16] H. Dai and H. Poor, "Asymptotic spectral efficiency of multicell MIMO systems with frequency-flat fading," IEEE Trans. Signal Processing, vol. 51, no. 11, pp. 2976-2988, Nov 2003.

[17] V. Marčenko and L. Pastur, "Distributions of eigenvalues of some sets of random matrices," Math. USSR-Sb., vol. 1, pp. 507-536, 1967. 